\title{
BCATI Overexpression Promotes Proliferation, Invasion, and Wnt Signaling in Non-Small Cell Lung Cancers
}

This article was published in the following Dove Press journal: OncoTargets and Therapy

\author{
Xiumin $\operatorname{Lin}^{1,2}$ \\ Shutao $\operatorname{Tan}^{3}$ \\ Lin $\mathrm{Fu}^{1,2}$ \\ Qianze Dong ${ }^{1,2}$
}

'Department of Pathology, College of Basic Medical Science, China Medical University, Shenyang, People's Republic of China; ${ }^{2}$ Department of Pathology, The First Affiliated Hospital of China Medical University, Shenyang, China; ${ }^{3}$ Department of Urology, Shengjing Hospital of China Medical University, Shenyang, People's Republic of China
Correspondence: Qianze Dong Department of Pathology, College of Basic Medical Science, China Medical University, Shenyang, People's Republic of China

Email dongqianze@hotmail.com
Introduction: Dysregulation of BCAT1 has been implicated in carcinogenesis. However, its clinical significance and biological roles in human non-small cell lung cancer (NSCLC) are not clear.

Methods: Immunohistochemistry was used to examine the protein expression of BCAT1 in 107 cases of lung cancer tissues. Biological roles and potential mechanisms of BCAT1 were examined using MTT, colony formation assay, Matrigel invasion assay, Western blot, RNAsequencing, and luciferase reporter assay.

Results: We found BCAT1 was upregulated in 60 of 107 lung cancer tissues and correlated with nodal metastasis, advanced stages and short overall survival. The Cancer Genome Atlas (TCGA) and ONCOMINE data analyses also indicated that BCAT1 was elevated in human NSCLC tissues. BCAT1 protein was higher in lung cancer cell lines than in normal bronchial epithelial cell line. BCAT1 overexpression increased the cell growth rate, colony numbers and invasion abilities in both BEAS-2B and H1299 cell lines, while BCAT1 siRNA decreased the cell proliferation rate, colony numbers, and inhibited invasion. RNA-sequencing (RNA-seq) and Gene Set Enrichment Analysis (GSEA) analyses indicated that BCAT1 overexpression activated Wnt/Myc signaling. Western blot revealed that BCAT1 increased protein expression of MMP7, cyclin D1, c-Myc, and decreased E-cadherin and p27 in the BEAS-2B and H1299 cell lines. Further experiments showed that BCAT1 overexpression elevated Wnt reporter luciferase activity and increased activate $\beta$-catenin protein while downregulating $\mathrm{p}$ - $\beta$-catenin protein expression. BCAT1 knockdown showed the opposite effects. TCGA data analysis suggested positive correlations between BCAT1 and c-Myc, cyclin D1, and MMP7 mRNA. Blockage of Wnt signaling using an inhibitor (ICG-001) downregulated c-Myc, cyclin D1, MMP7 expressions and abolished the upregulating effects of BCAT1 on these proteins.

Conclusion: In summary, our data showed that BCAT1 was overexpressed in human NSCLCs. BCAT1 facilitated cell proliferation and invasion possibly through regulation of the Wnt signaling pathway.

Keywords: BCAT1, lung cancer, Wnt, proliferation, c-Myc

\section{Introduction}

Non-small cell lung cancer (NSCLC) is a common malignancy worldwide and is one of the leading causes of cancer-related death. Its prognosis remains poor during the past decades despite novel surgical and chemotherapeutic advances. ${ }^{1}$ The prognoses of patients with NSCLC mainly correlate with malignant growth and metastasis, so it is important to identify effective targets involved in progression and invasion of NSCLCs. ${ }^{2,3}$ 
BCAT1 is a cytosolic and mitochondrial branched chain aminotransferases. This enzyme catalyzes the reversible transamination of branched-chain alpha-keto acids to branched-chain L-amino acids essential for cell growth., ${ }^{4,5}$ Overexpression of BCAT1 has been associated with a variety of cancers including glioblastoma, ${ }^{6}$ breast cancer, ${ }^{7}$ acute myeloid leukemia, ${ }^{8}$ chronic myeloid leukaemia, ${ }^{9}$ and gastric cancer. ${ }^{10}$ BCAT1 overexpression regulates amino acids metabolism and promotes malignant proliferation of cancer cells including cancer stem cells. ${ }^{8}$ BCAT1 also triggers epithelial-mesenchymal transition (EMT) in hepatocellular carcinoma. ${ }^{11}$ These studies indicate that BCAT1 is a potential oncoprotein in cancer progression. However, its clinical significance and biological roles in human NSCLCs remain unclear.

In the present study, we evaluated the clinical significance of BCAT1 protein in lung cancer tissues and examined the biological roles of BCAT1 in lung cancer cell lines. We also investigated the possible underlying mechanisms.

\section{Materials and Methods}

\section{Specimens}

The study protocol was approved by the institutional reviewer board of China Medical University. Lung cancer specimens were obtained from patients diagnosed with lung cancer patients between 2009 and 2013. Written informed consent was provided by the patients. The study was conducted in accordance with the Declaration of Helsinki. Clinical data including diagnosis and tumor grade were extracted from medical records. The histological diagnoses were evaluated for sections stained with hematoxylin and eosin (H\&E) according to the World Health Organization (WHO) classification guidelines.

\section{Immunohistochemistry}

Paraffin sections $(4 \mu \mathrm{m})$ were deparaffinized using xylene and treated with graded alcohol. Antigen retrieval was performed using citrate buffer ( $\mathrm{pH}$ 6.0). Sections were then incubated with normal goat serum. Then, sections were treated with BCAT1 antibody (1:400 dilution, Proteintech, USA) overnight at $4^{\circ} \mathrm{C}$. Immunohistochemical staining was performed using the Elivision Super Kit (MaiXin, Fuzhou, China). Staining was developed with DAB Plus Kit (MaiXin, Fuzhou, China).

The slides were evaluated by 2 pathologists. Cytoplasmic staining was regarded as positive staining. Intensity was scored as 0 (no/weak staining), 1 (moderate staining), and 2 (strong staining). Percentage scores were classified as 1:1-$25 \%, 2: 26-50 \% 3: 51-75 \%$ and $4: 76-100 \%$. Intensity and percentage scores were multiplied to obtain the final score. BCAT1 was considered low expression when the score was $<4$, and high expression when the score was $\geq 4$.

\section{Cell Culture and Transfection}

The normal human bronchial epithelial (NHBE) cell line BEAS-2B and lung cancer cell lines H1299, H1650, H460, A549, and H3255 were obtained from the American Type Culture Collection (Manassas, VA, USA). Cells were maintained in RPMI-1640 with $10 \%$ fetal bovine serum (FBS) (Gibco, USA). Empty vector and pCMV6-BCAT1 were from Origene and transfected into cells using Lipofectamine 3000 (Invitrogen, USA). siGENOME siRNA for BCAT1 (Dharmacon, USA) was used for knockdown experiments using Dharmafect1 reagent (Dharmacon, USA).

\section{Western Blotting}

Protein samples were separated by SDS-PAGE and then transferred to a PVDF membrane, then incubated with primary antibodies against BCAT1 (1:1000; Porteintech), cyclin D1, E-cadherin, MMP7, p- $\beta$-catenin, $\beta$-catenin, active $\beta$-catenin (1:1000, Cell Signaling, USA), and GAPDH (1:2000; Cell Signaling, USA) overnight. After incubation with secondary HRP conjugated antibodies (1:2000, Santa Cruz, USA). The Western blot bands were visualized using Pierce HRP substrate and recorded using DNR Bio-Imager.

\section{Quantitative Real-time PCR (SYBR Green Method)}

RNA was extracted using RNAiso reagent from TaKaRa (Dalian, China). Reverse transcription was performed using TaKaRa RT kit. Real-time PCR was conducted using the SYBRGreen Mastermix (TaKaRa) with ABI7500 PCR System (ABI, USA). $\beta$-actin was used as endogenous control.

\section{MTT Assay}

A total of 2000 cells were seeded in a total of five 96-well plates $24 \mathrm{hrs}$ after transfection. After $24 \mathrm{hrs}$ culture, $20 \mu \mathrm{L}$ MTT (thiazolyl blue) solution was added to the wells in the first plate. After 4 hrs of incubation, the medium was discarded and remaining formazan was dissolved using $150 \mu \mathrm{L}$ of dimethyl sulphoxide (DMSO). The plate was scanned using a plate reader at $550 \mathrm{~nm}$. The five plates were measured for a total of 5 days. 


\section{Colony Formation Assay}

In the colony formation assay, $24 \mathrm{hrs}$ after transfection, 1000 cells were seeded in $6-\mathrm{cm}$ dishes. These cells were cultured for approximately 2 weeks. The dishes were washed with PBS and stained with Giemsa. The number of colonies with more than 50 cells was counted.

\section{Matrigel Invasion Assay}

Matrigel invasion assay was performed using Coring Transwell chamber coated with $20 \mu \mathrm{L}$ Matrigel from BD bioscience. Cells were placed in the upper chamber with serum-free medium. The lower chamber was placed with medium with $10 \%$ serum. After $24 \mathrm{~h}$ incubation, the cells invading through the membrane were fixed and stained with hematoxylin.

\section{RNA-Sequencing}

RNA was extracted from NHBE and NHBE-BCAT1 cells using RNAiso reagent from TaKaRa (Dalian, China). The RNA-sequencing (RNA-seq) was performed by Novogene corporation (Beijing, China). Gene Set Enrichment Analysis (GSEA) was conducted using the software downloaded from the GSEA website (http://software.broadinsti tute.org).

\section{Luciferase Reporter Assay}

Reporter gene luciferase assay was carried out in cells growing on a 24 well plate. These cells were co-transfected with the TOP-Flash firefly luciferase reporter $(0.2 \mu \mathrm{g})$ along with the Renilla luciferase reporter $(0.02 \mu \mathrm{g})$ for $18 \mathrm{hrs}$ using Lipofectamine 3000 (Invitrogen, USA). The luciferase activity was measured using a Dual-Luciferase Reporter Assay System (Promega, CA, USA). Read firefly and renilla luciferase signals were detected using Berthold Luminometer. Relative firefly/renilla signals were normalized as fold changes.

\section{Statistical Analysis}

SPSS version 16.0 (SPSS, Chicago, IL, USA) was used for statistical analyses. The BCAT1 mRNA data (RNA-seq) of NSCLCs were obtained from TCGA database. The BCAT1 mRNA data (microarray) of NSCLCs were obtained from ONCOMINE. The correlations between BCAT1 levels and clinicopathological factors were analyzed using the $\chi 2$ tests. Kaplan-Meier was used to estimate the probability of patient survival, and differences in the survival were compared by using Log-rank test. Cox regression model was used for multivariate and univariate analysis. Student's $t$-test and ANOVA were used to compare data obtained from biological experiments. A $p<0.05$ was considered as statistical significance.

\section{Results \\ BCATI Is Elevated in Non-Small Cell Lung Cancers (NSCLCs)}

We first analyzed the expression patterns of BCAT1 in 107 cases of paraffin embedded NSCLC tissues and 15 cases of normal lung tissues using immunohistochemistry. Weak/ negative staining of BCAT1 was found in normal bronchial epithelial cells (Figure 1A) and alveolar cells (Figure 1B). Increased cytoplasmic BCAT1 staining was found in 60 of $107(56.1 \%)$ the NSCLC tissues (Figure 1C-F). Table 1 shows that high expression of BCAT1 high expression was significantly associated with advanced TNM stage $(\mathrm{p}=0.0149)$ and nodal metastasis $(\mathrm{p}=0.0172)$. Kaplan-Meier survival analysis showed that patients with high BCAT1 had shorter overall survival compared to those with low BCAT1 expression ( $\mathrm{p}=0.0028$, Log-rank test, Figure $1 \mathrm{G}$ ). In addition, multivariate analysis using Cox regression model indicated that TNM stage and BCAT1 overexpression were independent, unfavorable prognostic factors (TNM stage: $p=0.0007$; BCAT1: $\mathrm{p}=0.0135$ ) (Table 2).

We next analyzed the BCAT1 mRNA expression of NSCLC tissues and normal lung tissues using data from the ONCOMINE database. The Okayama lung dataset, Beer lung dataset, Su lung dataset, Landi lung dataset and Hou lung dataset of ONCOMINE showed that BCAT1 mRNA was significantly higher in lung adenocarcinoma compared with normal lung tissues (Figure $2 \mathrm{~A}-\mathrm{C}, \mathrm{F}$ and $\mathrm{G}$ ). The Hou lung dataset showed that BCAT1 mRNA was also higher in lung squamous cell carcinoma than in normal lung tissues (Figure 2H). In addition, the Weiss lung dataset showed that the DNA copy number was higher in lung adenocarcinoma and squamous cell carcinoma compared with that in normal lung tissues (Figure 2D and E).

The Cancer Genome Atlas (TCGA) lung cancer cohort indicated that the overall survival of patients with high BCAT1 mRNA was worse than those with low expression. (Log-rank test, $p<0.01$, Figure $2 \mathrm{I}$ ). Collectively, these data indicated that BCAT1 was upregulated and its levels were correlated with malignant features in human NSCLCs.

\section{BCATI Promotes Cell Proliferation and Invasion}

We profiled BCAT1 protein and mRNA in the BEAS-2 B normal cell line and a panel of lung cancer cell lines: 
A

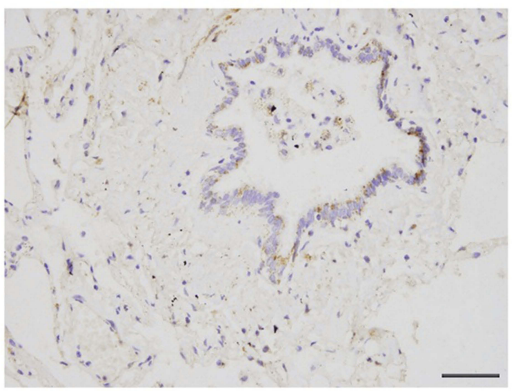

C

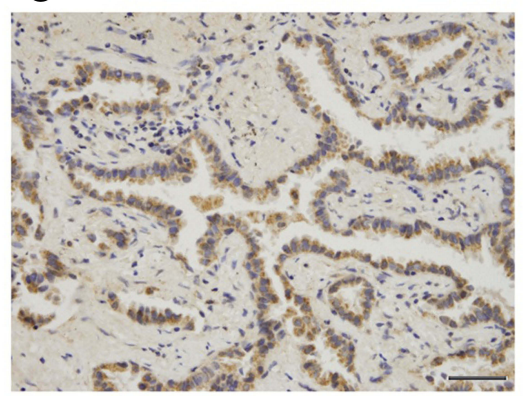

E

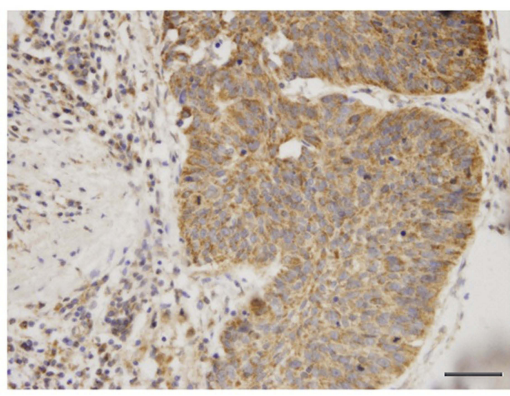

B

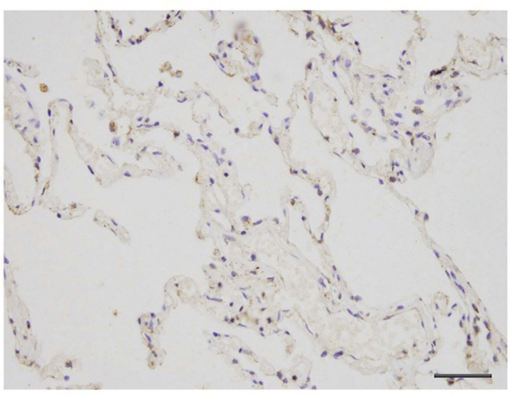

D

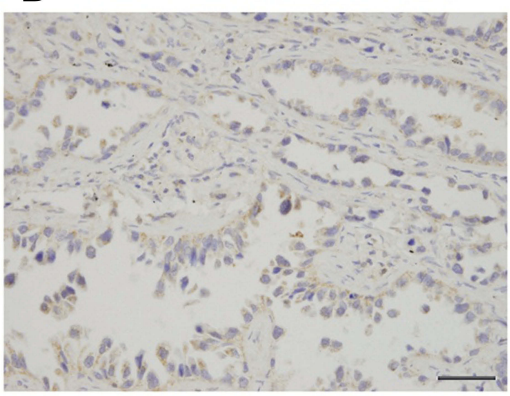

F

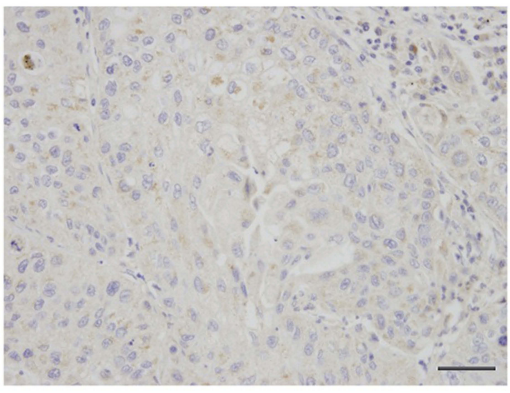

G

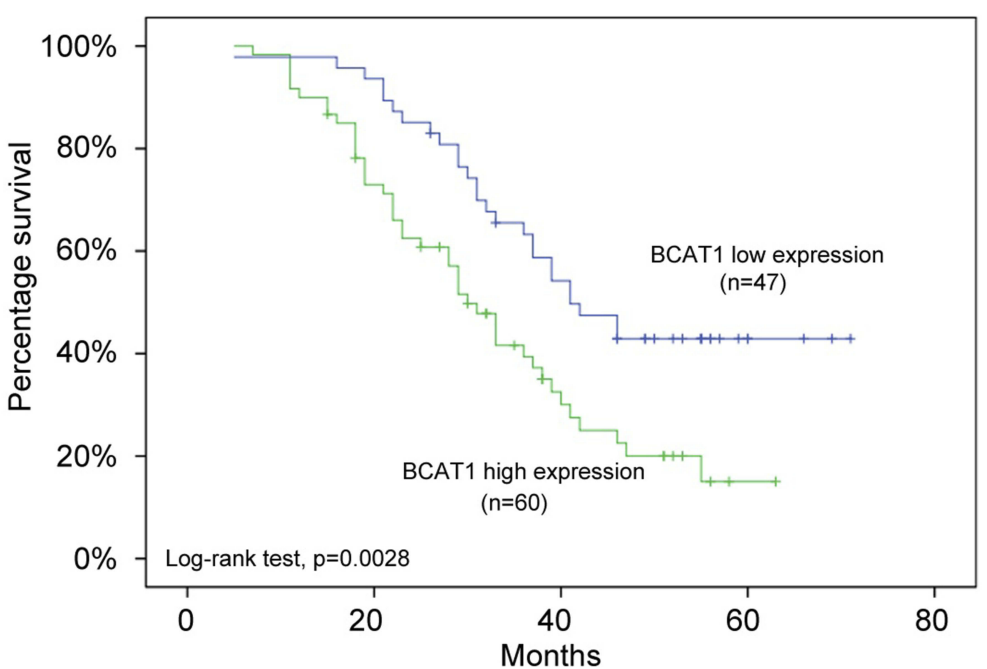

Figure I BCATI expression is increased in lung cancer tissues. (A) Negative/weak immunostaining of BCATI in normal bronchial epithelial tissue. (B) Weak BCATI immunostaining in normal alveolar epithelial tissue. (C) Positive cytoplasmic BCATI expression in a case of lung adenocarcinoma. (D) Weak cytoplasmic BCATI immunostaining in a case of lung adenocarcinoma. (E) Strong cytoplasmic BCATI expression in a case of lung squamous cell carcinoma. (400x; bars indicate 50 $\mu \mathrm{m}$ ). (F) Negative/weak cytoplasmic BCATI expression in a case of lung squamous cell carcinoma. (G) Overall survival curve of lung cancer patients with high and low BCATI expression (Log-rank test, $p=0.0028$ ). 
Table I Distribution of BCATI Status in NSCLC According to Clinicopathological Characteristics

\begin{tabular}{|c|c|c|c|c|}
\hline Characteristics & $\begin{array}{l}\text { Number } \\
\text { of } \\
\text { Patients }\end{array}$ & $\begin{array}{l}\text { BCATI } \\
\text { Low } \\
\text { Expression }\end{array}$ & $\begin{array}{l}\text { BCATI } \\
\text { High } \\
\text { Expression }\end{array}$ & $P$ \\
\hline \multicolumn{5}{|l|}{ Age } \\
\hline$<60$ & 58 & 23 & 35 & 0.3329 \\
\hline$\geq 60$ & 49 & 24 & 25 & \\
\hline \multicolumn{5}{|l|}{ Gender } \\
\hline Male & 63 & 29 & 34 & 0.5993 \\
\hline Female & 44 & 18 & 26 & \\
\hline \multicolumn{5}{|l|}{ Differentiation } \\
\hline Well & 37 & 17 & 20 & 0.7595 \\
\hline Moderate- Poor & 70 & 30 & 40 & \\
\hline \multicolumn{5}{|l|}{ Histology } \\
\hline Adenocarcinoma & 59 & 23 & 36 & 0.2534 \\
\hline $\begin{array}{l}\text { Squamous cell } \\
\text { carcinoma }\end{array}$ & 48 & 24 & 24 & \\
\hline \multicolumn{5}{|l|}{ TNM Stage } \\
\hline I & 45 & 27 & 17 & 0.0149 \\
\hline II & 31 & 11 & 20 & \\
\hline III & 31 & 9 & 22 & \\
\hline \multicolumn{5}{|l|}{ Tumor Status } \\
\hline $\mathrm{TI}$ & 32 & 11 & 21 & 0.1935 \\
\hline T2-T4 & 75 & 36 & 39 & \\
\hline \multicolumn{5}{|l|}{ Nodal Metastasis } \\
\hline Negative & 59 & 32 & 27 & 0.0172 \\
\hline Positive & 48 & 15 & 33 & \\
\hline
\end{tabular}

Table 2 Univariate and Multivariate Analysis for Predictive Factors in Patients with NSCLC

\begin{tabular}{|l|l|l|l|l|}
\hline & \multicolumn{2}{|l|}{ Univariate } & \multicolumn{2}{l|}{ Multivariate } \\
\hline Factors & $\begin{array}{l}\text { Hazard Ratio } \\
(95 \% \text { Cl) }\end{array}$ & p value & $\begin{array}{l}\text { Hazard Ratio } \\
(95 \% \text { Cl) }\end{array}$ & p value \\
\hline Gender & $\begin{array}{l}1.047 \\
(0.650-1.686)\end{array}$ & 0.8499 & & \\
\hline Histology & $\begin{array}{l}1.173 \\
(0.723-1.888)\end{array}$ & 0.5120 & & \\
\hline Differentiation & $\begin{array}{l}1.144 \\
(0.824-1.587)\end{array}$ & 0.4233 & & \\
\hline TNM stage & $\begin{array}{l}1.845 \\
(1.372-2.481)\end{array}$ & 0.0001 & $\begin{array}{l}1.727 \\
(1.261-2.364)\end{array}$ & 0.0007 \\
\hline BCATI & $\begin{array}{l}2.063 \\
(1.261-3.377)\end{array}$ & 0.004 & $\begin{array}{l}1.902 \\
(1.142-3.168)\end{array}$ & 0.0135 \\
\hline
\end{tabular}

including H1299, H1650, H460, A549, H3255 (Figure 3A and B). The H460, A549 and H3255 cell lines had rela- tively high BCAT1 mRNA and protein expressions compared with the normal BEAS-2B cell line.

BCAT1 overexpression was measured in the BEAS-2B and H1299 cell lines. BCAT1 siRNA knockdown was performed in the A549 cell line. The transfection efficiency was confirmed by RT-qPCR and Western blot (Figure 3C). MTT assay was used to detect change of cell growth rate for 5 days after $48 \mathrm{hrs}$ of transfection. MTT assay showed that BCAT1 overexpression increased proliferation rate in both BEAS-2B and H1299 cell lines while BCAT1 siRNA decreased proliferation rate in A549 cell line (Figure 4A). Colony formation showed that BCAT1 overexpression significantly increased colony numbers in both BEAS-2B and H1299 cell lines while BCAT1 depletion decreased colony numbers in A549 cell line (Figure 4B). In addition, Matrigel invasion assay showed that BCAT1 overexpression increased invading cell numbers while BCAT1 knockdown decreased invading cell numbers in lung cancer cell lines (Figure 4C).

\section{BCATI Regulates Wnt Signaling and Wnt Target Proteins}

To further elucidate the underlying mechanisms of BCAT1, we overexpressed BCAT1 in BEAS-2B cells and used RNA-sequencing to profile the global mRNA change induced by BCAT1 (Supplementary Table 1). GSEA revealed an enrichment for Myc target genes, cyclin D1 target genes, and Wnt target genes (Figure 5A). Because Myc and cyclinD1 were both downstream targets of Wnt signaling pathway, we hypothesized that BCAT1 might activate Wnt signaling in BEAS-2B and lung cancer cells. Using Western blot analysis we showed that BCAT1 overexpression upregulated protein levels of cyclinD1, c-Myc and MMP7, while it downregulated p27 and E-cadherin in both BEAS-2B and H1299 cell lines (Figure 5B). BCAT1 depletion showed the opposite effects in A549 cell line.

We also determined $\mathrm{p}-\beta$-catenin (inactive) and active $\beta$ catenin levels by Western blot and Wnt activity by luciferase reporter assay. We found that BCAT1 overexpression upregulated active $\beta$-catenin and downregulated inactive p- $\beta$-catenin in both BEAS-2B and H1299 cell lines. TopFlash Luciferase reporter assay showed that BCAT1 overexpression enhanced Wnt signaling activity while BCAT1 depletion showed the opposite effects (Figure 5C). Thus, 
A

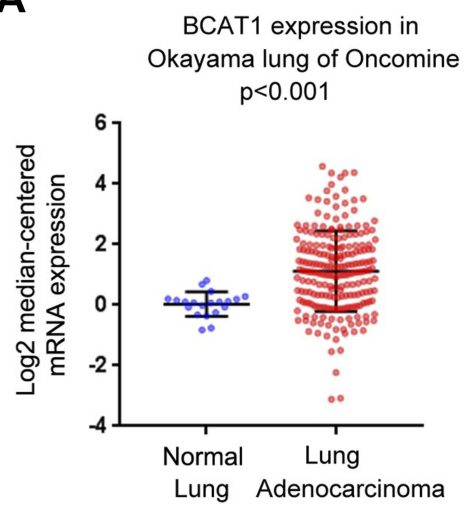

D
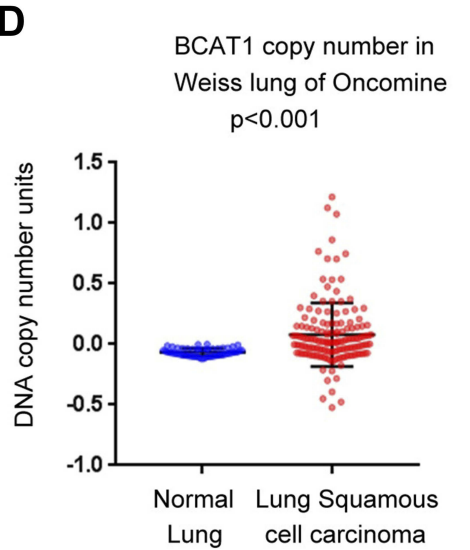

G

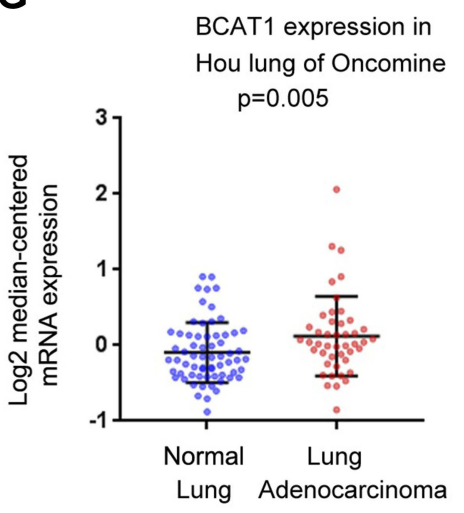

B

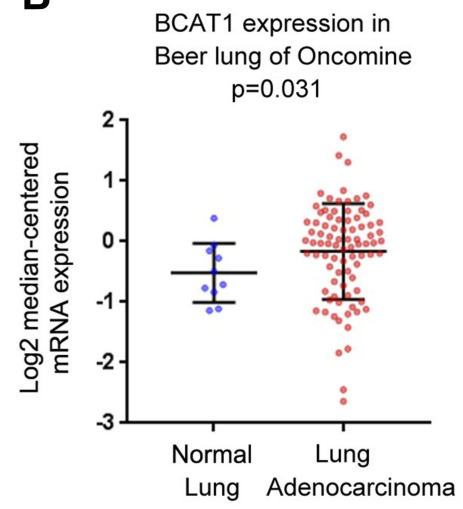

E

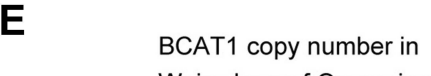

Weiss lung of Oncomine $p=0.044$

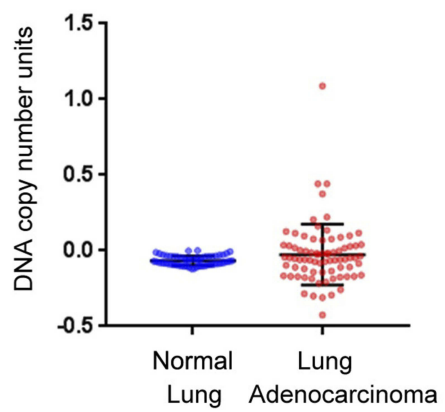

H

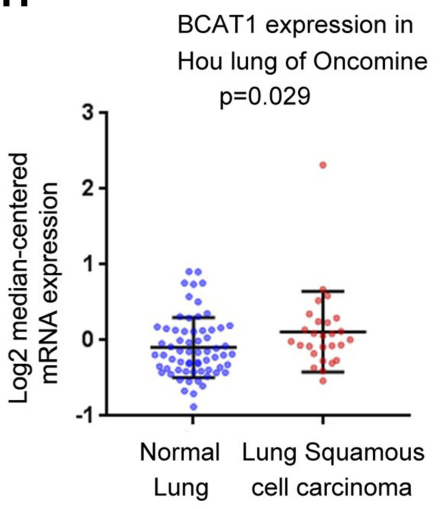

C
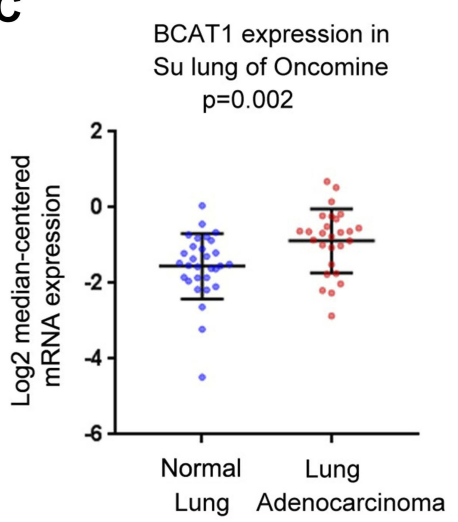

F

BCAT1 expression in Landi lung of Oncomine $p=0.015$
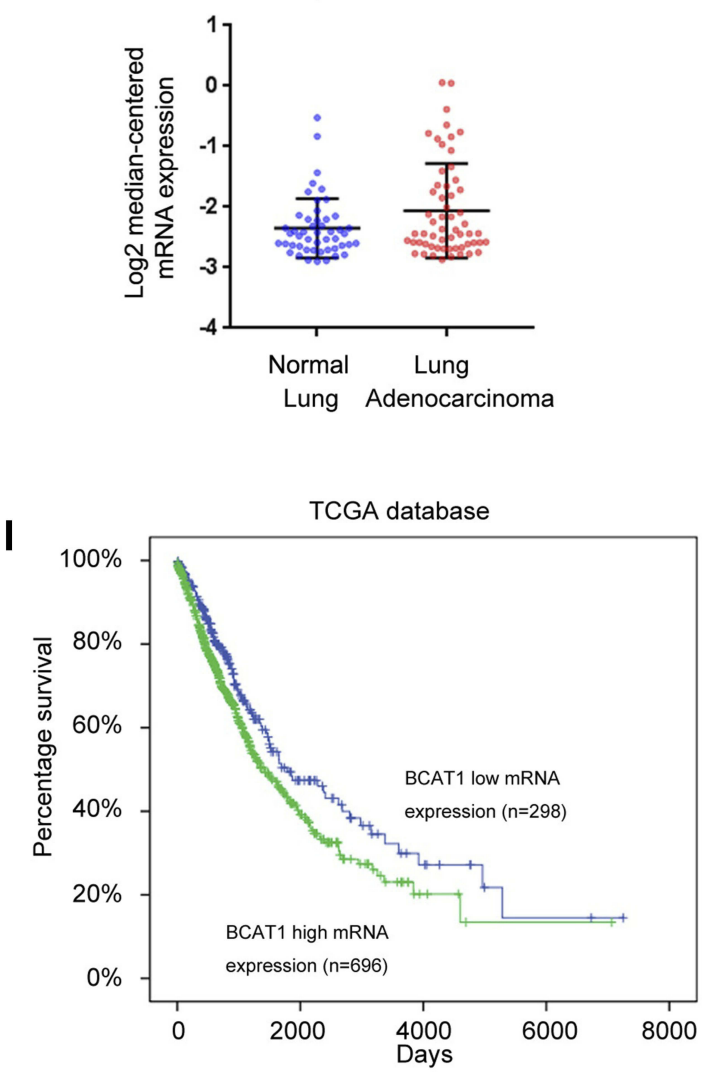

Figure 2 BCATI expression in NSCLC tissues using ONCOMINE microarray and TCGA databases. (A-H) Analysis of the lung cancer dataset of ONCOMINE. BCATI mRNA and DNA copy number were significantly higher in lung cancer tissues compared with normal lung tissues. (I) Overall survival curve of lung cancer patients with high and low BCATI expression using TCGA dataset (Log-rank test, $\mathrm{p}<0.0 \mathrm{I}$ ).

the impact of BCAT1 on lung cancer cell proliferation and invasion may depend on $\mathrm{Wnt} / \beta$-catenin pathway.

\section{BCATI Regulates Cyclin DI, MMP7 and c-Myc Through Wnt Signaling Pathway}

To confirm the link of BCAT1 with Wnt signaling and its target genes in lung cancer tissues, we further analyzed
mRNA of 515 cases of lung cancer from TCGA. Figure 6A indicates that Pearson's correlation showed significant correlations between BCAT1 mRNA and c-Myc, cyclin D1, MMP7, and TCF4 mRNA, supporting our results that BCAT1 was a positive regulator of Wnt signaling.

To further validate the interaction between Wnt signaling and BCAT1, we used $\backslash$ Wnt inhibitor ICG-001 in BEAS-2B and 
A

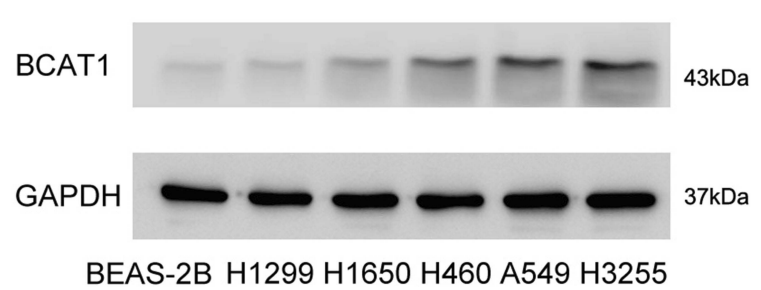

B

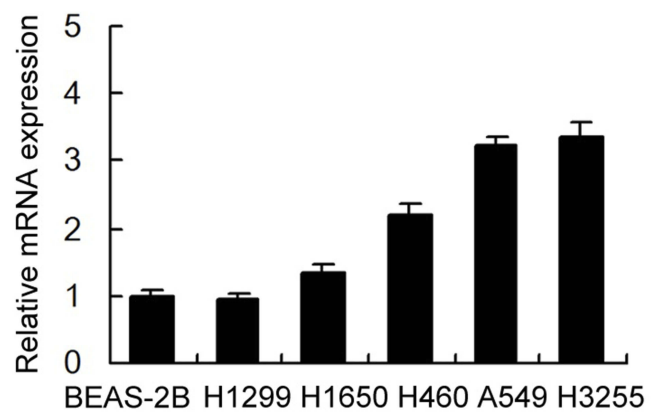

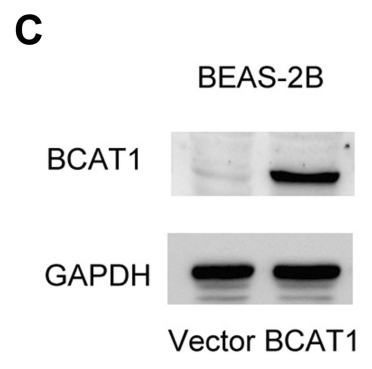
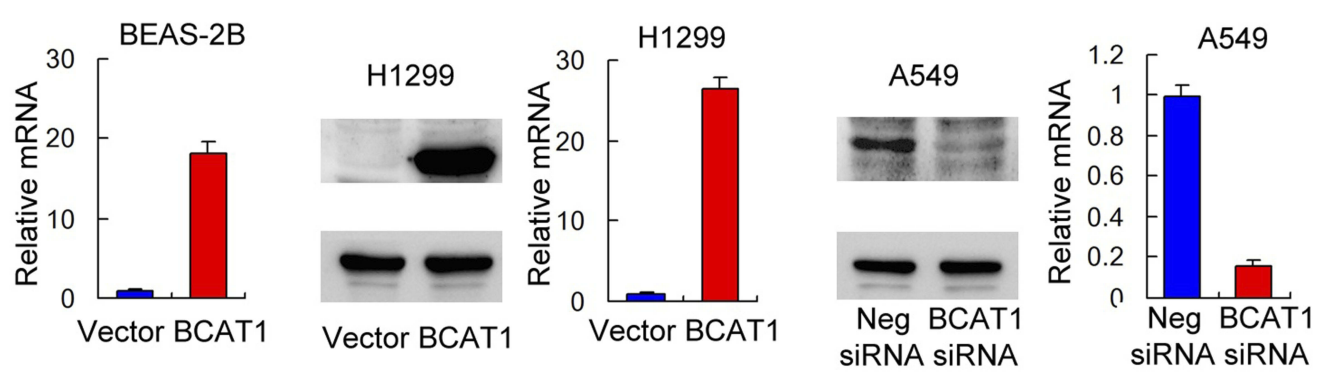

Figure 3 BCATI expression in lung cancer cell lines and transfection efficiency. (A) Protein expression of BCATI in normal BEAS-2B cell line and lung cancer cell lines (HI299, A549, H460, HI650 and H3255). (B) mRNA expression of BCATI in normal BEAS-2B cell line and lung cancer cell lines. (C) Plasmid transfection significantly upregulated BCATI protein and mRNA levels in BEAS-2B and HI299 cell lines while siRNA treatment downregulated BCATI protein and mRNA expression in A549 cell line.

H1299 cells transfected with BCAT1 plasmid. The effect of ICG-001 was confirmed using Top-Flash Luciferase reporter assay (Figure 6B). Wnt inhibitor significantly downregulated levels of cyclin D1, MMP7 and c-Myc. Wnt inhibitor also slightly downregulated BCAT1 protein. There was significant amelioration of BCAT1-induced upregulation of cyclin D1, MMP7 and c-Myc, implying that effects of BCAT1 upon these protein were dependent upon Wnt signaling. Together, these results suggested that Wnt played a central role during BCAT1-induced increase of cyclin D1, MMP7 and c-Myc in lung cancer cells.

\section{Discussion}

The main finding of the present study is that BCAT1 is upregulated in human non-small cell lung cancer tissues and markedly accelerated cell growth and invasion through activation of Wnt signaling. As the role of BCAT1 on carcinogenesis mainly focused on its regulation on branched-chain amino acids (BCAA) metabolism in previous studies, ${ }^{9,12}$ its impact on other signaling pathways has not been fully investigated. We believe our results showed, for the first time, the involvement of Wnt pathway in the biological effect of BCAT1 on lung cancer development.

Recent evidence has suggested BCAT1 upregulation in various cancers. $^{7-11}$ However, its clinical significance in
NSCLCs remains unclear. Our current results demonstrated that elevation of BCAT1 protein correlated nodal metastasis and higher TNM stage, which was further supported by TCGA and ONCOMINE data analyses. Importantly, we showed that high BCAT1 status was associated with poor patient prognosis and serves as an independent prognostic factor, suggesting it could serve as a clinical biomarker for the malignant behavior of NSCLC.

To explore its biological roles, we overexpressed BCAT1 in normal BEAS-2B cell line and cancerous H1299 cell line. We also knocked down BCAT1 in A549 cell line using siRNA. MTT and colony formation assays showed that BCAT1 positively regulated proliferation in normal cancer lung cancer cells. Accordingly, Matrigel invasion assay showed that BCAT1 also promoted cell invasion. Mechanistically, GESA analysis using RNA-seq data indicated that BCAT1 overexpression activated Myc and cyclin D1 target genes, which was confirmed by Western blot analysis. Both c-Myc and cyclin D1 are important oncogenes involving lung carcinogenesis and proliferation. ${ }^{13,14}$ It should be noted that although BCAA metabolism plays a part in growth support of lung cancer cells, other pathways induced by BCAT1 may also contribute to malignant progression. A recent report showed that BCAT1 was able to induce EMT process in hepatocellular carcinoma cells, ${ }^{11}$ which was 
A
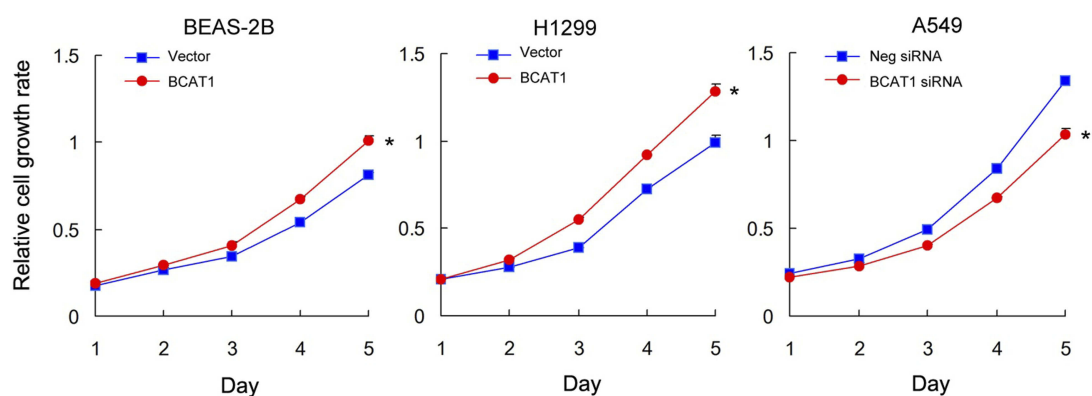

B
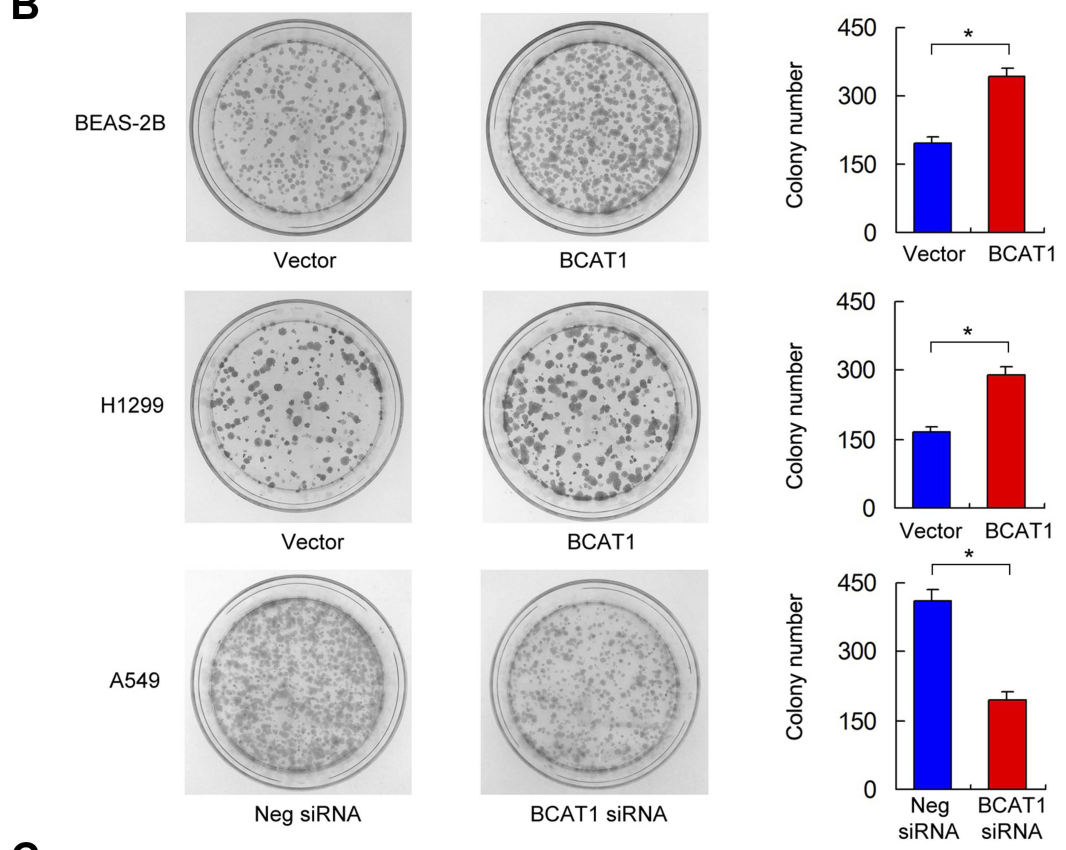

C
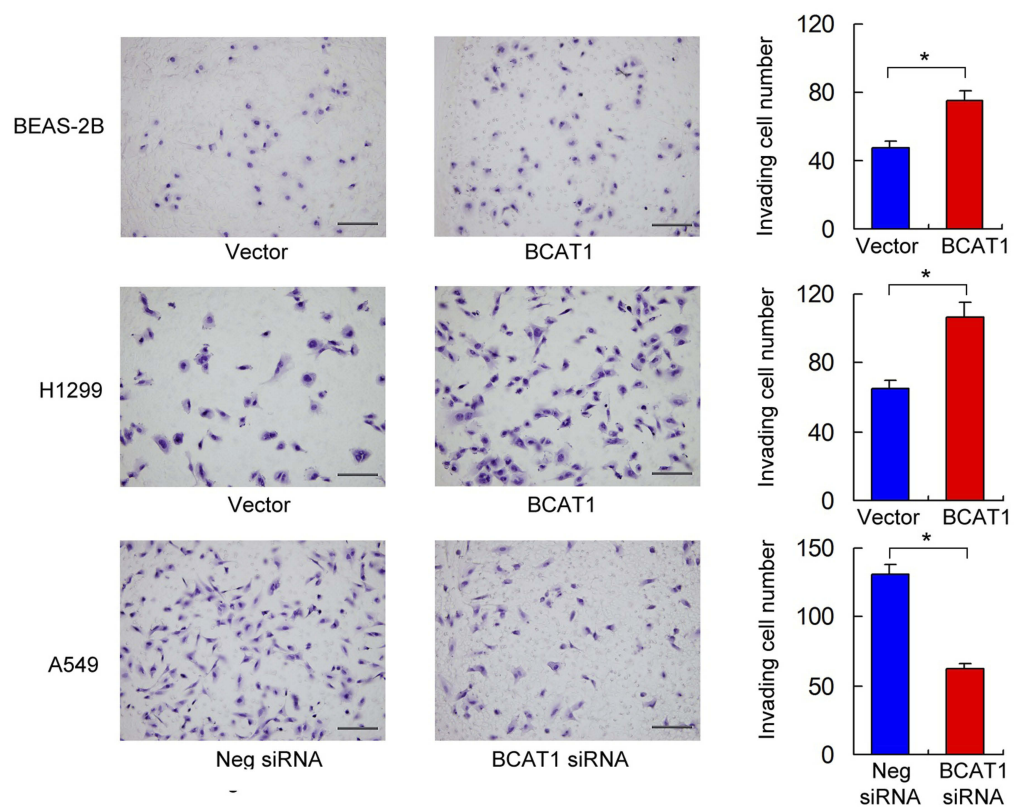

Figure 4 BCATI regulates proliferation, colony formation and invasion. (A) MTT assay showed that BCATI overexpression promoted proliferation rate while BCATI depletion inhibited proliferation rate. ${ }^{*} \mathrm{p}<0.05$ (Student's t-test and ANOVA test) (B) Colony formation assay showed that BCATI overexpression upregulated colony number while BCATI depletion decreased colony number. ${ }^{*} p<0.05$. (Student's $t$-test) (C) Matrigel invasion assay showed that BCATI overexpression increased invading cell number while BCATI depletion decreased invading cell number. ${ }^{*} \mathrm{p}<0.05$. (Student's $t$-test) (bars indicate $50 \mu \mathrm{m}$ ). 
A
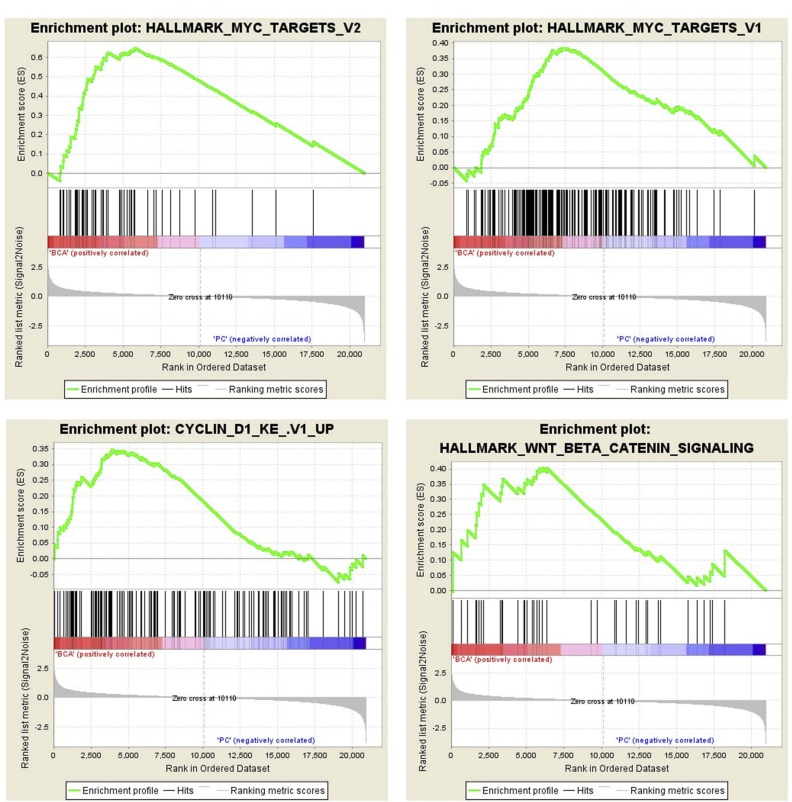

B

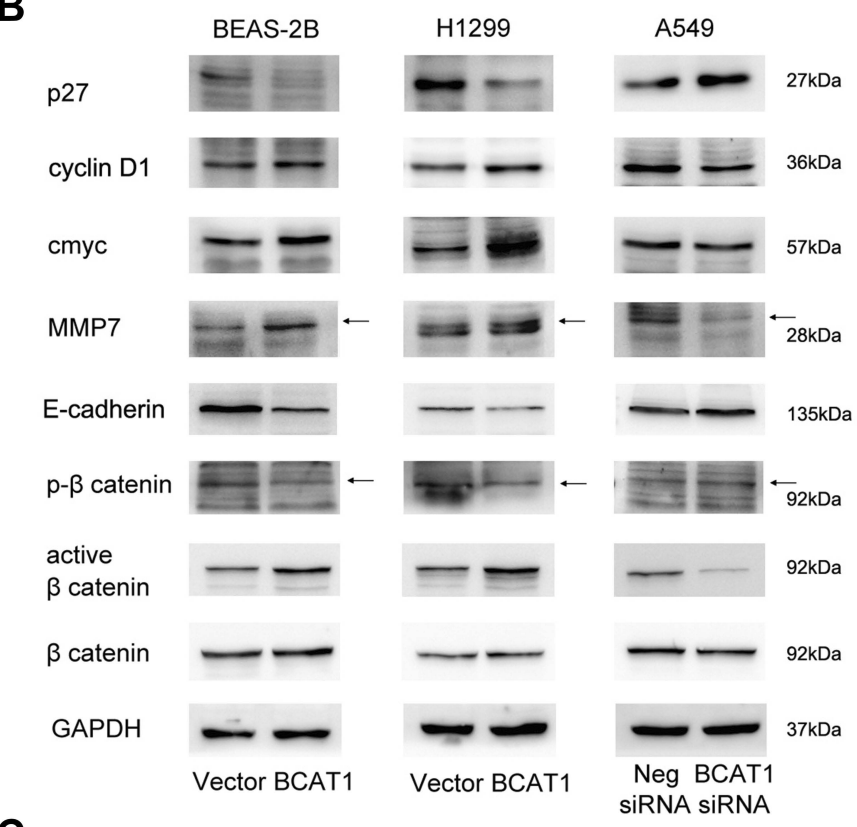

C

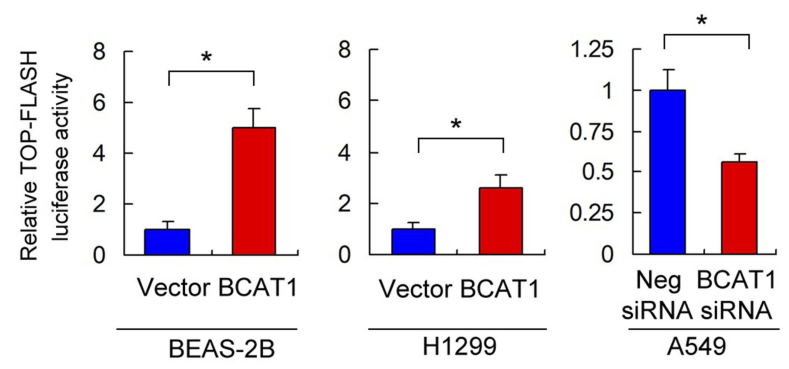

Figure 5 BCATI regulates Wnt signaling and targets. (A) RNA-sequencing and Gene set expression analysis (GSEA) revealed an enrichment for Myc target genes, cyclin DI target genes, and Wnt target genes in BEAS-2B cells with BCATI overexpression. (B) Western blot analysis demonstrated that BCATI overexpression upregulated protein levels of cyclin DI, c-Myc, MMP7, active $\beta$-catenin and downregulated p27, E-cadherin and p- $\beta$-catenin in BEAS-2B and HI299 cell lines. In contrast, BCATI depletion downregulated cyclin DI, c-Myc, MMP7, active $\beta$-catenin and upregulated p27, E-cadherin and p- $\beta$-catenin in A549 cell line. (C) Top-Flash Luciferase reporter assay showed that BCATI overexpression increased Wnt signaling activity in BEAS-2B and HI 299 cell lines. BCATI depletion decreased Wnt signaling activity in A549 cell line. *P $<0.05$. (Student's $t$-test). Arrows indicated the specific Western blot bands. 
A

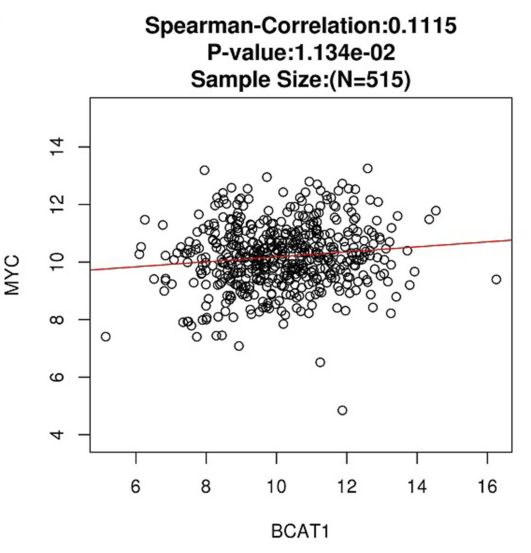

Spearman-Correlation:0.138

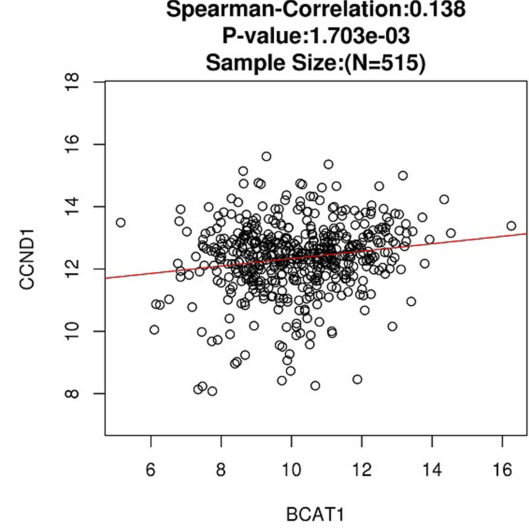

B

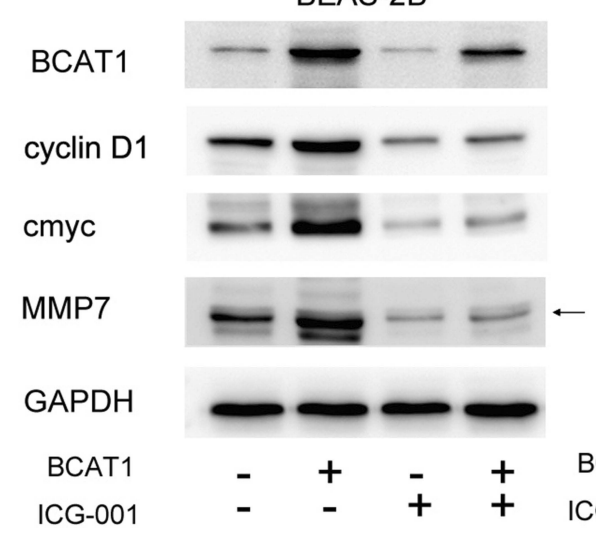

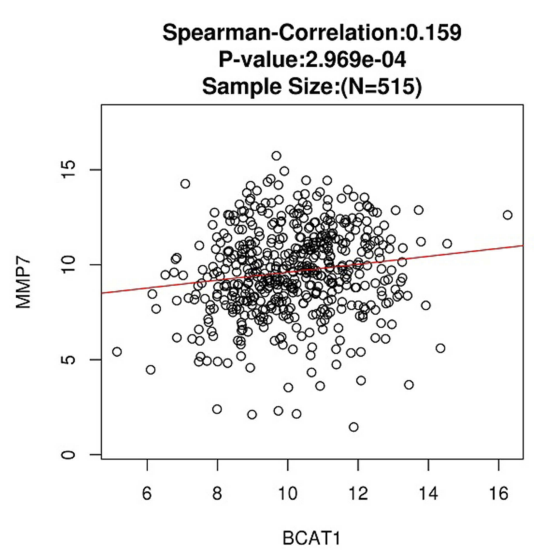

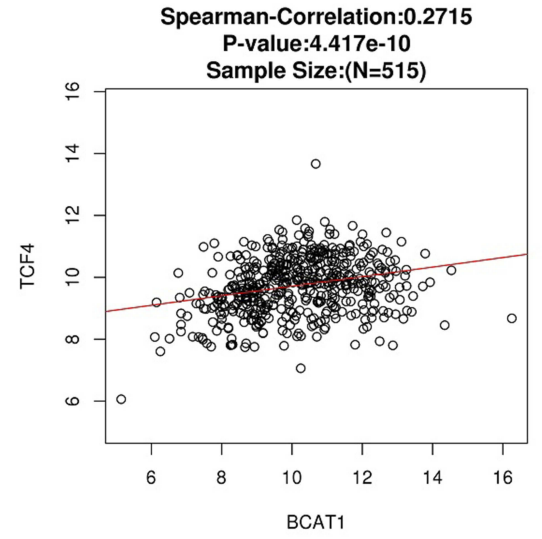

H1299
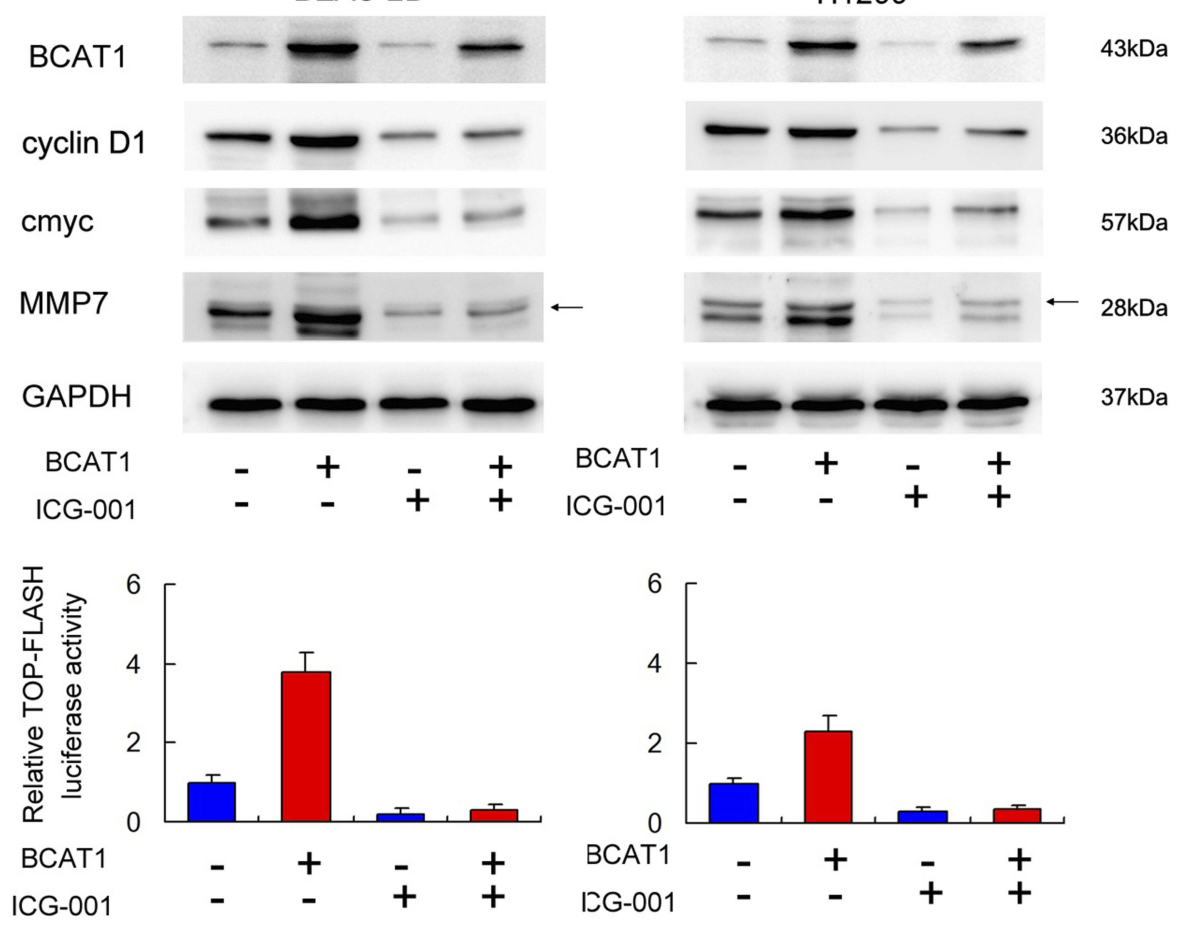

Figure 6 BCATI regulates cyclin DI, MMP7, c-Myc through Wnt signaling. (A) Pearson's correlation analysis showed positive associations between BCATI and cyclin DI, MMP7, c-Myc and TCF4 mRNA in 515 cases of lung cancer. (B) Wnt inhibitor ICG-00I was used in cells transfected with BCATI plasmid or control vector. ICG-00I decreased cyclin DI, MMP7, c-Myc protein expression. In cells treated with ICG-00I, the effect of BCATI on cyclin DI, MMP7 and c-Myc protein was significantly ameliorated. Top-Flash Luciferase reporter assay showed that ICG-00I decreased Wnt signaling activity in BEAS-2B and HI299 cell lines. 
also confirmed in our study using BEAS-2B and lung cancer cell lines. Our data, therefore, provided some evidences for the novel role of BCAT1 in NSCLC proliferation. MMP-7 has many biological functions like tumor invasion and metastasis. ${ }^{15}$ Tumors with high MMP7 break down the extracellular matrix, leading to invasion and metastasis. ${ }^{16}$ In NSCLCs, MMP-7 also highly correlates with cancer proliferation. ${ }^{17}$ Our result showed BCAT1 overexpression upregulated MMP7 while downregulated E-cadherin, which partly explained its positive regulation of lung cancer invasion.

Additionally, GSEA indicated that BCAT1 overexpression activated $\mathrm{Wnt} /$ beta-catenin signaling, which was further validated using Wnt specific TopFlash luciferase reporter assay. This was consistent with our observation that BCAT1 positively regulated c-Myc, cyclin D1 and MMP7 levels as it is well established that these were targets of Wnt signaling pathway. ${ }^{18}$ BCAT1 overexpression upregulated active $\beta$ catenin while downregulated inactive phospho- $\beta$-catenin, supporting the role of BCAT1 on Wnt pathway. To further confirm the BCAT1-Wnt axis, we used an inhibitor to block Wnt function and the effects of BCAT1 on cyclin D1, c-Myc and MMP7 were significantly reduced. Analyses of TCGA database also supported the positive correlations of BCAT1 mRNA with cyclin D1, c-Myc, MMP7 and TCF4. These findings suggest a link between BCAT1, Wnt and c-Myc/ cyclin D1/MMP7 during lung cancer growth and invasion. Notably, a previous report showed c-Myc could directly bind to the specific promoter region of BCAT1 gene. ${ }^{19}$ Our result showed that BCAT1 expression was slightly decreased after treatment with Wnt inhibitor. These results are consistent with a positive feedback loop between BCAT1 and c-Myc, providing a new mechanism involving the interaction of BCAT1 and Wnt/c-Myc interplay in lung cancers.

In conclusion, the current study revealed a novel role of BCAT1 in NSCLC by linking its oncogenic characteristics with Wnt signaling pathway. Our data provide insight into the mechanisms of BCAT1 in cancer progression, indicating the potential therapeutic strategy of targeting BCAT1-Wnt/c-Myc axis. Further studies are needed to fully characterize the mechanism of Wnt regulation by BCAT1.

\section{Acknowledgment}

The study was supported by The National Natural Science Foundation of China (No. 81772468) to Qianze Dong.

\section{Disclosure}

We declare that we have no conflicts of interest in this work.

\section{References}

1. Siegel RL, Miller KD, Jemal A. Cancer statistics, 2017. CA Cancer J Clin. 2017;67(1):7-30. doi:10.3322/caac.21387

2. Dong QZ, Wang Y, Tang ZP, et al. Derlin-1 is overexpressed in non-small cell lung cancer and promotes cancer cell invasion via EGFR-ERK-mediated up-regulation of MMP-2 and MMP-9. Am J Pathol. 2013;182(3):954-964. doi:10.1016/j.ajpath.2012.11.019

3. Dong QZ, Wang Y, Dong XJ, et al. CIP2A is overexpressed in non-small cell lung cancer and correlates with poor prognosis. Ann Surg Oncol. 2011;18(3):857-865. doi:10.1245/s10434-010-1313-8

4. Eden A, Benvenisty N. Involvement of branched-chain amino acid aminotransferase (Bcat1/Eca39) in apoptosis. FEBS Lett. 1999;457 (2):255-261. doi:10.1016/S0014-5793(99)01054-6

5. Ben-yosef T, Eden A, Benvenisty N. Characterization of murine BCAT genes: bcat1, a c-Myc target, and its homolog, Bcat2. Mamm Genome. 1998:9(7):595-597. doi:10.1007/s003359900825

6. Tonjes M, Barbus S, Park YJ, et al. BCAT1 promotes cell proliferation through amino acid catabolism in gliomas carrying wild-type IDH1. Nat Med. 2013;19(7):901-908. doi:10.1038/nm.3217

7. Zhang L, Han J. Branched-chain amino acid transaminase 1 (BCAT1) promotes the growth of breast cancer cells through improving mTOR-mediated mitochondrial biogenesis and function. Biochem Biophys Res Commun. 2017;486(2):224-231. doi:10.1016/j. bbrc.2017.02.101

8. Raffel S, Falcone M, Kneisel N, et al. BCAT1 restricts alphaKG levels in AML stem cells leading to IDHmut-like DNA hypermethylation. Nature. 2017;551(7680):384-388. doi:10.1038/nature24294

9. Hattori A, Tsunoda M, Konuma T, et al. Cancer progression by reprogrammed BCAA metabolism in myeloid leukaemia. Nature. 2017;545(7655):500-504. doi:10.1038/nature22314

10. Xu Y, Yu W, Yang T, et al. Overexpression of BCAT1 is a prognostic marker in gastric cancer. Hum Pathol. 2018;75:41-46. doi:10.1016/j. humpath.2018.02.003

11. Qi LN, Xiang BD, Wu FX, et al. Circulating tumor cells undergoing EMT provide a metric for diagnosis and prognosis of patients with hepatocellular carcinoma. Cancer Res. 2018;78(16):4731-4744.

12. Gu Z, Liu Y, Cai F, et al. Loss of EZH2 reprograms BCAA metabolism to drive leukemic transformation. Cancer Discov. 2019;9 (9):1228-1247. doi:10.1158/2159-8290.CD-19-0152

13. Bragelmann J, Bohm S, Guthrie MR, Mollaoglu G, Oliver TG, Sos ML. Family matters: how MYC family oncogenes impact small cell lung cancer. Cell Cycle. 2017;16(16):1489-1498. doi:10.1080/ 15384101.2017.1339849

14. Zhang LQ, Jiang F, Xu L, et al. The role of cyclin D1 expression and patient's survival in non-small-cell lung cancer: a systematic review with meta-analysis. Clin Lung Cancer. 2012;13(3):188-195. doi:10.1016/j.cllc.2011.10.003

15. Merchant N, Nagaraju GP, Rajitha B, et al. Matrix metalloproteinases: their functional role in lung cancer. Carcinogenesis. 2017;38 (8):766-780. doi:10.1093/carcin/bgx063

16. Wilson CL, Matrisian LM. Matrilysin: an epithelial matrix metalloproteinase with potentially novel functions. Int J Biochem Cell Biol. 1996;28(2):123-136. doi:10.1016/1357-2725(95)00121-2

17. Liu D, Nakano J, Ishikawa S, et al. Overexpression of matrix metalloproteinase-7 (MMP-7) correlates with tumor proliferation, and a poor prognosis in non-small cell lung cancer. Lung Cancer. 2007;58(3):384-391. doi:10.1016/j.lungcan.2007.07.005

18. Stewart DJ. Wnt signaling pathway in non-small cell lung cancer. J Natl Cancer Inst. 2014;106(1):djt356. doi:10.1093/jnci/djt356

19. Zhou W, Feng X, Ren C, et al. Over-expression of BCAT1, a c-Myc target gene, induces cell proliferation, migration and invasion in nasopharyngeal carcinoma. Mol Cancer. 2013;12:53. doi:10.1186/ $1476-4598-12-53$ 


\section{Publish your work in this journal}

OncoTargets and Therapy is an international, peer-reviewed, open access journal focusing on the pathological basis of all cancers, potential targets for therapy and treatment protocols employed to improve the management of cancer patients. The journal also focuses on the impact of management programs and new therapeutic agents and protocols on patient perspectives such as quality of life, adherence and satisfaction. The manuscript management system is completely online and includes a very quick and fair peer-review system, which is all easy to use. Visit http://www.dovepress.com/ testimonials.php to read real quotes from published authors.

Submit your manuscript here: https:/www.dovepress.com/oncotargets-and-therapy-journal 